\title{
Accompagnement du parcours de formation personnalisé
}

Yves Girmens

\section{OpenEdition}

1 Journals

Édition électronique

URL : http://journals.openedition.org/trema/1472

DOI : 10.4000/trema.1472

ISSN : 2107-0997

Éditeur

Faculté d'Éducation de l'université de Montpellier

\section{Édition imprimée}

Date de publication : 1 janvier 2003

Pagination : 179-185

ISSN : 1167-315X

\section{Référence électronique}

Yves Girmens, « Accompagnement du parcours de formation personnalisé », Tréma [En ligne], 20-21 | 2003, mis en ligne le 01 avril 2003, consulté le 19 avril 2019. URL : http:// journals.openedition.org/trema/1472 ; DOI : 10.4000/trema.1472

Ce document a été généré automatiquement le 19 avril 2019.

Trema 


\title{
Accompagnement du parcours de formation personnalisé
}

\author{
Yves Girmens
}

\section{Problématique}

1 La formation professionnelle des professeurs-stagiaires des collèges et lycées se réalise à travers des actions de formation communes à tous les stagiaires et d'autres qui prennent en compte la dimension personnelle de chaque stagiaire.

Les modules de formation disciplinaire (à contenu didactique) et de formation générale sont des passages obligés pour tous les stagiaires où la différenciation ne peut être marquée car ces formations sont dispensées de manière collective.

Le mémoire, le parcours personnalisé, le projet d'aide au travail et à l'insertion des élèves (dans le cadre de la formation générale), la première visite à visée formative, constituent des démarches de formation où l'aspect personnel de la formation est dominant.

Pour qu'une formation professionnelle au métier d'enseignant soit efficace, il est nécessaire qu'elle s'appuie sur une action volontaire de l'enseignant en formation.

Il s'agit de permettre à chaque stagiaire, de mettre en résonance les divers aspects personnels de sa formation mais aussi de piloter le plus possible son processus de formation, en identifiant des besoins qui lui sont propres, en cherchant des réponses appropriées à ces besoins et en s'engageant personnellement dans les actions de formation.

\section{Le projet d'accompagnement}

\subsection{Le contenu}

2 Le projet a consisté à organiser le parcours de formation de chaque professeur-stagiaire, dans ses composantes personnelles, en lui offrant la possibilité de s'appuyer sur un 
groupe d'échanges constitué de professeurs stagiaires comme lui, accompagné par un formateur.

Le rôle du formateur était d'assurer un suivi personnalisé de la formation des stagiaires du groupe dont il a la charge, en permettant aux stagiaires de faire des liens entre les divers aspects de la formation, en favorisant l'expression de besoins individuels et en aidant à la recherche de réponses personnelles à ces besoins.

Ce suivi personnalisé s'est décliné suivant deux axes, d'une part, une relation entre chaque stagiaire et le tuteur de suivi, qui s'est traduit par des rencontres autour de sujets déterminés par l'évolution du stagiaire, d'autre part, l'insertion dans un groupe, qui s'est concrétisée par des séances de travail coopératif autour de questions liées à la formation à l'enseignement des mathématiques.

L'expérience a concerné 16 professeurs-stagiaires en mathématiques de collège et lycée et trois formateurs.

\subsection{La mise en œuvre}

3 Le projet s'est réalisé par une alternance de rencontres entre le tuteur et chaque stagiaire et de séances de travail collectif rassemblant les stagiaires (chaque groupe contenait de quatre à six stagiaires).

Les séances d'entretien individuel ont comme objectif de faire émerger les besoins propres de chacun, de définir les actions à mener alors que les séances collectives ont comme but de favoriser un partage d'expériences, une mutualisation des projets d'actions et de leur réalisation, ainsi qu'une régulation sur les choix effectués.

Le parcours de formation a été scindé en trois grandes périodes qui ont des fonctions distinctes.

4 La première période avait comme objectif d'établir un profil de chaque stagiaire, à partir des informations issues du récit de ses premières expériences et de l'expression de ses attentes.

C'est dans cette phase qu'a eu lieu une visite formative, menée par le formateur, qui a permis à chaque stagiaire d'identifier de premiers objets de travail.

En effet, à l'issue de l'analyse de la séance conduite par le formateur, en s'appuyant sur les observations d'autres stagiaires invités, il a été demandé à chaque stagiaire de rédiger un rapport dans lequel il doit mettre en évidence les aspects principaux qu'il a retenus ainsi que les points sur lesquels il décide de mener un travail, avec l'aide de son tuteur de stage.

Cela constitue alors l'amorce d'un plan de formation personnel du stagiaire.

$\mathrm{Au}$ cours de cette première étape, une première séance collective a été organisée pour permettre à chaque stagiaire de raconter ses premières expériences, d'évoquer ses premières réussites, de formuler ses premières questions, et d'exprimer ses premières envies en matière de formation; le formateur enregistre alors ces différents éléments et, en s'appuyant sur les échanges du groupe, provoque des explicitations et des mises en commun.

Puis, au cours d'un entretien individuel avec chaque stagiaire, le formateur l'a amené à préciser les questions qu'il se pose, à formuler les raisons du choix des objectifs de travail à l'issue de la visite formative, à expliciter ce qu'il espère découvrir ou apprendre dans telle action qu'il a envie de mener, et le cas échéant à formuler une idée de sujet de mémoire. 
6 Le but de cette étape a été de permettre au stagiaire de commencer à construire son projet de formation.

Par exemple, à un stagiaire qui manifestait l'envie d'aller voir des séances d'enseignement des mathématiques en ZEP, le formateur a demandé ce qu'il souhaite apprendre à travers cette action et à quelles questions il espère apporter des réponses.

Cela a permis au stagiaire, dans un premier temps, d'exprimer son angoisse de devoir un jour enseigner en ZEP, de dire son besoin de se rassurer par rapport à cette perspective puis, dans un deuxième temps, d'avouer qu'il veut se rendre compte s'il est possible d'enseigner des mathématiques à des élèves en difficulté et qu'il veut savoir comment gérer une classe d'élèves démotivés ; il a ainsi reconnu qu'il se posait cette question dans sa propre classe.

A l'issue de cette phase, compte-tenu des divers éléments dégagés, le formateur a demandé au stagiaire de produire un écrit présentant, d'une part, les actions qu'il envisage de mener ainsi que les questions auxquelles il pense que ces actions fourniront une réponse et, d'autre part, les options possibles pour ces actions, ainsi que des modalités envisagées.

C'est à ce moment-là que, en fonction de l'action envisagée, le formateur a pu inciter le stagiaire à rechercher des opportunités dans son environnement immédiat: investigations auprès d'équipes de professeurs de son établissement, repérage de stages d'établissement.

7 La deuxième période a visé à permettre à chaque stagiaire de mettre sur pied son projet et de le mettre en œuvre. Les séances de travail ont eu pour but, une fois certains acquis professionnels reconnus, d'identifier de nouveaux besoins et de prendre des décisions sur les actions à mener.

$\mathrm{Au}$ cours d'une séance collective, en présence du formateur, chaque stagiaire a fait une présentation du projet qu'il a ébauché.

Le formateur, en sollicitant des réactions des autres participants et en stimulant l'échange entre eux, les a aidés à affiner leur projet et à mieux définir sa mise en œuvre.

C'est l'occasion pour le formateur d'inciter les stagiaires à mener certaines actions ensemble - le travail à plusieurs est considéré comme une priorité - de suggérer de nouvelles idées d'actions, de nouvelles pistes de ressources à explorer.

8 Lors de cette séance, un temps a été prévu pour que chaque stagiaire expose l'avancement de sa réflexion par rapport au mémoire professionnel (choix du sujet, problématique...), le rôle des autres étant de l'amener, en lui posant les questions nécessaires, à étayer son sujet par rapport à l'origine de son choix et la faisabilité de son étude.

Quand il l'a estimé possible, le formateur a incité les stagiaires à relier le travail sur le mémoire et certaines actions envisagées.

$\mathrm{Au}$ cours d'un entretien individuel avec chaque stagiaire, le formateur a aidé le stagiaire à mettre sur pied un plan d'actions qui peuvent être de natures diverses: initiatives individuelles, actions avec le concours d'une tierce personne (le tuteur de stage par exemple) ou l'insertion dans des actions instituées (équipes de recherche, stage existant...).

L'entretien a commencé par un temps où le stagiaire a été invité à exposer ce qu'il juge réussi et à peu prés acquis dans sa pratique, puis à faire un bilan des actions qu'il a menées avec le concours de son tuteur de stage. 
Ensuite, le formateur, en s'efforçant de mettre en relation toutes les informations dont il dispose sur le stagiaire (récit du professeur, observation de sa pratique, éléments fournis par tuteur de stage...), a examiné avec lui les actions qu'il envisage et l'a conduit à mieux définir son projet, voire à le compléter.

La rencontre s'est achevée par un temps de synthèse des actions projetées et par une prévision de leur réalisation : description des actions, des dispositifs, des modalités...

$\mathrm{Au}$ cours de la troisième période, les professeurs stagiaires ont été amenés en premier lieu, à faire un bilan de l'avancement de leurs projets et en second lieu, à partager leurs expériences et leurs premières conclusions.

C'est au cours d'une séance collective, en présence du formateur, que les stagiaires ont été invités à présenter un compte-rendu des projets réalisés, en mettant en évidence les difficultés rencontrées, les premiers enseignements tirés et les prolongements envisagés.

Lors de cette séance, le rôle du formateur a consisté à faire étudier l'adéquation entre les conclusions, les réponses obtenues et les attentes initiales puis à suggérer des réajustements et à impulser des relances.

Ensuite, en entretien individuel, le formateur a fait expliciter au stagiaire ce qu'il pense avoir tiré de ses expériences par rapport à sa pratique d'enseignement et, en fonction de l'évolution du stagiaire et des besoins constatés, lui a fait des suggestions de nouvelles actions ou de renouvellements d'expériences.

Le suivi du parcours de formation s'est conclu par une séance collective au cours de laquelle chacun a dressé un bilan des actions de formation qu'il a menées (y compris le mémoire), en mettant l'accent sur le bénéfice qu'il pense en avoir retiré par rapport aux questions initiales.

\section{Analyse}

11 Pour analyser les effets de ce projet, nous retenons trois axes: l'inscription dans un groupe d'accompagnement, la personnalisation de la formation et le suivi du parcours de formation.

\subsection{Le groupe d'accompagnement}

12 L'inscription dans un groupe de pairs a favorisé la co-construction de connaissances professionnelles que chacun peut ensuite s'approprier; en ce sens, elle a contribué de façon décisive à l'émergence d'une identité professionnelle des stagiaires.

Les séances de partages d'expériences, de mutualisation des questions, la recherche coopérative de réponses ont été vécues par les stagiaires comme très positives: ils se disent à la fois rassurés et stimulés dans leur action de formation, découvrant que les autres rencontrent des problèmes semblables et se posent les mêmes questions qu'eux.

\subsection{Une dimension de personnalisation de la formation}

Lors de l'expérimentation, il a été relevé une modification du rapport des stagiaires à la formation : ils ont exprimé le sentiment d'être reconnus, de ne pas se sentir jugés, d'être pris en compte dans leurs questions et attentes propres.

La plupart des actions projetées ont pu être menées à bien, parfois en autonomie, seuls ou à plusieurs, parfois avec l'étayage d'un formateur (par regroupement de stagiaires en 
atelier), mais certaines actions projetées et identifiées par des stagiaires comme nécessaires n'ont pu être réalisées devant certaines difficultés matérielles (l'encadrement ou le contexte n'ont parfois pu être trouvés).

Bon nombre d'actions envisagées par les stagiaires étaient voisines et ont pu être regroupées au sein de projets communs à plusieurs stagiaires, ce qui a favorisé leur coopération sur une même action.

Des actions telles que participer à des séances d'enseignement dans des classes spécifiques (ZEP ou SEGPA), aller observer une séance en CM2, travailler avec un conseiller d'éducation ont pu ainsi être mises sur pied en travail coopératif, ce qui a nécessité de rechercher une structure d'accueil et une personne - ressource.

D'autres projets, plus individuels, tels qu'une recherche autour de dispositifs d'aide aux élèves, un travail sur la voix et le positionnement en classe, une réflexion sur la prise de parole en classe, le montage d'un projet d'intégration de TICE, ont demandé un étayage plus important.

Il apparaît qu'au delà de la réalisation ou non de certaines actions, c'est le processus dans lequel chaque stagiaire est engagé par ce dispositif qui a été fructueux.

En effet, c'est la phase d'élaboration d'un projet qui est apparue très formatrice et un projet d'action, même s'il n'a pas abouti, est le résultat d'un processus dans lequel chaque stagiaire a développé une attitude réflexive sur lui-même et sur ses pratiques.

En outre, le dispositif a favorisé, pour un nombre significatif de stagiaires, la recherche d'une cohérence dans les actions de formation, à partir de besoins identifiés.

\subsection{Le suivi du parcours de formation}

16 Il s'est avéré important qu'un formateur dispose du maximum d'informations sur la pratique d'enseignement d'un stagiaire qu'il accompagne.

C'est pourquoi, il semble nécessaire que le formateur chargé du suivi des parcours de formation d'un groupe de stagiaires mène lui-même les visites formatives des stagiaires dont il a la charge, ce qui n'a pu être toujours le cas, en raison de contraintes horaires.

Le suivi du parcours de formation d'un stagiaire nécessite, dans tous les cas, une liaison continue entre le formateur concerné et le tuteur du stage en responsabilité de ce stagiaire, afin de permettre au formateur d'avoir une connaissance aussi précise que possible de la pratique d'enseignement du stagiaire.

17 Le rôle du formateur chargé du suivi est, en s'appuyant sur les attentes déclarées des stagiaires et des informations dont il dispose, de permettre à chaque stagiaire de prendre conscience de ses besoins.

Il doit veiller à laisser à chaque stagiaire l'initiative du choix des actions à mener ainsi que des modalités; il doit tenir le plus grand compte de l'évolution du stagiaire, ce qui suppose qu'il se mette à son écoute et qu'il suscite un réajustement constant de son projet personnel.

\section{Conclusion}

18 Ce dispositif permet d'entrevoir qu'il est possible, de favoriser, d'une manière raisonnable, un engagement des professeurs stagiaires dans leur processus de formation, en leur permettant de s'appuyer sur un groupe d'échanges et de coopération, en leur 
laissant la responsabilité de certaines actions et les stimulant dans leur choix.

Un tel dispositif, bien que léger (quatre séances collectives, trois entretiens individuels, $20 \mathrm{~h}$ assurées par le formateur responsable d'un groupe), semble assurer pour les stagiaires une meilleure dévolution des actions de formation professionnelle, du fait qu'ils ont le sentiment de prendre davantage part à leur formation et qu'ils sont conduits à établir des liens entre les diverses formations auxquelles ils participent.

Ce projet permet aussi de favoriser, à travers des actions communes, un travail coopératif entre plusieurs stagiaires, ce qui peut être considéré comme un objectif important de la formation des enseignants aujourd'hui.

Enfin, il s'avère nécessaire, pour qu'une telle action de formation soit viable et reproductible, d'une part, de susciter la participation de formateurs volontaires et motivés et d'autre part, de pouvoir disposer d'un réseau de personnes-ressources, dans divers domaines concernant l'enseignement, susceptibles d'accueillir et d'encadrer des stagiaires pour des actions de formation qu'ils ont eux-mêmes décidé de mener.

\section{RÉSUMÉS}

Le projet avait comme objectif de permettre à chaque professeur stagiaire de vivre la formation professionnelle comme un parcours de formation, dans lequel il peut prolonger les différentes actions de formation imposées par l'institution par des actions qu'il décide de mener à partir de la perception d'attentes et de besoins personnels.

Pour cela, chaque stagiaire est inséré dans un groupe de pairs qui est encadré par un formateur. La mission du formateur est tout à la fois de favoriser un travail coopératif d'autoformation au sein du groupe et d'accompagner chaque stagiaire de manière individualisée pour l'aider à identifier ses besoins de formation propres et à trouver des réponses appropriées à ces besoins.

This research project had as its objective making it possible for each and every teacher trainee to experience his professional training as a training journey in which he can fulfill and pursue the various training actions imposed by the training institute through actions he may decide to follow on the basis of his perception of expectations and personal needs.

To this end, each trainee is enrolled in a group of peers which is supervised by a training instructor.

The mission of the training instructor is both to create the favourable conditions for cooperative self-training within the group and to accompany each student teacher on an individual basis in order to help him to identify his unique training needs and to seek out the solutions appropriate to his needs.

INDEX

Keywords : accompaniment, need, personal training

Mots-clés : accompagnement, besoin, coopération, formation personnalisée 


\section{AUTEUR}

\section{YVES GIRMENS}

Professeur, IUFM de Montpellier 